\title{
On the pairing structure of the vacuum induced by a magnetic field in $2+1$-dimensional Dirac field theory
}

\author{
Giovanni Jona-Lasinio* \\ Dipartimento di Fisica, Università di Roma "La Sapienza" \\ P.le Aldo Moro, 2 - 00185 Roma \\ Francesca Maria Marchetti ${ }^{\dagger}$ \\ Scuola Normale Superiore \\ P.zza dei Cavalieri, 7 - 56126 Pisa \\ Roma, 3rd October 2017
}

\begin{abstract}
Using a sequence of Bogoliubov transformations, we obtain an exact expression for the vacuum state of a Dirac field in $2+1$ dimensions in presence of a constant magnetic field. This expression reveals a peculiar two level pairing structure for any value of the mass $m \geq 0$. This calculation clarifies the nature of the condensate in the lowest Landau level whose existence has been emphasized recently by several authors.
\end{abstract}

\section{Introduction}

The phenomenon of symmetry breaking induced by an external homogeneous magnetic field of an internal $U(2)$ symmetry for the Dirac field in $2+1$ dimensions has recently attracted some attention. The reason why people are interested in this phenomenon is its possible relevance in the more complex context of interacting fields, especially in situations where spontaneous symmetry breaking is expected, like in strong coupling QED and NJL models [7, 9, 10, 6].The symmetry breaking is connected with a property of the theory called spectral asymmetry of the lowest Landau level (LLL) and it is revealed by the calculation of the order parameter

$$
\lim _{m \rightarrow 0}\left\langle B\left|\bar{\psi}^{B}(x) \psi^{B}(x)\right| B\right\rangle=-\frac{|e B|}{2 \pi}
$$

\footnotetext{
* Email: Jona@roma1.infn.it

$\dagger$ Email: fmarchet@cibs.sns.it
} 
where $m$ is the mass of the Dirac field. This parameter is sometimes called flavor condensate.

The expression (11) can be obtained using the proper time method of Schwinger 11] or through the development the propagator in terms of Landau poles [5, 7]. From the calculations one sees that the result (11) depends on the dominance of the LLL, when $m \rightarrow 0$.

The methods used to obtain (11) do not reveal completely the structure of the condensate since they do not need the explicit expression of the vacuum state. In this paper we obtain the explicit expression of the vacuum in presence of a magnetic field in terms of the creation and destruction operator of the free field $(B=0)$. The problem is not entirely trivial and is solved by performing in sequence two Bogoliubov transformations. The first leads

from the vacuum of the free field $|0\rangle$ to a BCS type state $\widehat{|0\rangle}$, pairing particles and antiparticles with opposite momenta. This step does not involve the magnetic field. The second transformation acts on auxiliary creation and destruction operators associated with $\widehat{|0\rangle}$. Their pairing leads to the vacuum $|B\rangle$ of the Dirac field in presence of magnetic field. These operators when applied to $\widehat{|0\rangle}$ create or destroy states having the same quantum numbers of the Landau levels; they can be interpreted as degenerate eigenstates of an auxiliary hamiltonian $\widehat{H}$ with energy equal to the LLL. The structure of the vacuum just described is the same for any value of $m$ including $m \rightarrow 0$ and disappears in the non relativistic limit that is for $m \rightarrow \infty$.

We believe that this calculation has a twofold interest. The problem of the Dirac field in $2+1$ dimensions in presence of a constant magnetic field is solved by operator techniques which make clear its non perturbative nature. In view of the study of non trivial models with interaction it provides an application of the principle solve the ground state first, then it is easier to understand the excitations 12].

\section{The Dirac equation in $2+1$ dimensions}

The main properties of the $2+1$ dimensional Dirac field theory in an external homogeneous magnetic field have been discussed in [3, 4, 2, 7] and will be now summarized to make the paper self-contained.

Consider the lagrangian $\mathcal{L}$ :

$$
\begin{aligned}
\mathcal{L} & =\bar{\psi}^{B}(x)\left[i \gamma^{\mu} \mathcal{D}_{\mu}-m\right] \psi^{B}(x) \\
\mathcal{D}_{\mu} & =\partial_{\mu}-i e A_{\mu}
\end{aligned}
$$

where $e$ is the modulus of the electron charge. Since the external magnetic 
field is constant and homogeneous we can choose the Landau gauge:

$$
A_{\mu}=-\delta_{\mu 1} B x^{2}
$$

In $2+1$ dimensions there are two inequivalent minimal versions of the Dirac algebra [2]:

$$
\begin{aligned}
& \left\{\gamma^{\mu}, \gamma^{\nu}\right\}=2 g^{\mu \nu} \quad \mu=0,1,2 \\
& \gamma^{0^{\dagger}}=\gamma^{0} \quad \gamma^{i \dagger}=-\gamma^{i} \quad i=1,2 \text {. }
\end{aligned}
$$

In the following we shall use the representations:

$$
\widetilde{\gamma}^{0}=\sigma_{3} \quad \widetilde{\gamma}^{1}=i \sigma_{1} \quad \widetilde{\gamma}^{2}=i \sigma_{2}
$$

and

$$
\widehat{\gamma}^{0}=-\sigma_{3} \quad \widehat{\gamma}^{1}=-i \sigma_{1} \quad \widehat{\gamma}^{2}=-i \sigma_{2}
$$

where $\sigma_{i}$ are the Pauli matrices. The complex spinors associated with the two minimal versions will be respectively indicated with $\psi_{1}(x)$ e $\psi_{2}(x)$.

A chiral version is obtained as the direct sum of the two inequivalent irreducible representations (5) and (6):

$$
\gamma^{\mu}=\left(\begin{array}{cc}
\widetilde{\gamma}^{\mu} & 0 \\
0 & \widehat{\gamma}^{\mu}
\end{array}\right)=\left(\begin{array}{cc}
\widetilde{\gamma}^{\mu} & 0 \\
0 & -\widetilde{\gamma}^{\mu}
\end{array}\right) \quad \psi(x)=\left(\begin{array}{l}
\psi_{1}(x) \\
\psi_{2}(x)
\end{array}\right) .
$$

With this notation the lagrangian in the chiral version is connected to the lagrangians in the two minimal versions by the following relationship:

$$
\begin{gathered}
\mathcal{L}=\mathcal{L}_{1}+\mathcal{L}_{2} \quad \mathcal{L}_{i}=\bar{\psi}_{i}^{B}(x)\left[i \widetilde{\gamma}^{\mu} \mathcal{D}_{\mu}-\alpha_{i} m\right] \psi_{i}^{B}(x) \\
\alpha_{i}=\left\{\begin{array}{ll}
1 & i=1 \\
-1 & i=2
\end{array} \quad \bar{\psi}_{i}^{B}(x)=\psi_{i}^{B^{\dagger}}(x) \sigma_{3} \quad \forall i=1,2 .\right.
\end{gathered}
$$

The two lagrangians $\mathcal{L}_{1}$ and $\mathcal{L}_{2}$ differ only for the sign of the mass term.

In the minimal versions both lagrangians $\mathcal{L}_{1}$ and $\mathcal{L}_{2}$ are invariant under charge conjugation $\mathcal{C}$, while the mass term changes sign under parity $\mathcal{P}$ and time reversal $\mathcal{T}$. In the minimal versions there is no generator of chiral symmetry, that is there is no symmetry (except $\mathcal{P}$ and $\mathcal{T}$ ), that distinguishes the theory with mass from the theory with zero mass.

In the chiral version the three discrete symmetries $\mathcal{C}, \mathcal{P}$ and $\mathcal{T}$ are preserved and therefore the mass term breaks neither parity nor time reversal. 
Besides, we can introduce the following matrices, which anticommute with each other and with the other matrices $\gamma^{\mu}$ defined in (7):

$$
\gamma^{3}=i\left(\begin{array}{ll}
0 & \mathbb{I} \\
\mathbb{I} & 0
\end{array}\right) \quad \gamma^{5}=i \gamma^{0} \gamma^{1} \gamma^{2} \gamma^{3}=i\left(\begin{array}{cc}
0 & \mathbb{I} \\
-\mathbb{I} & 0
\end{array}\right)
$$

In the chiral version we can introduce an internal $U(2)$ group of symmetry, defined by the generators $T_{\alpha}$ :

$$
\begin{aligned}
& U(\omega) \in U(2): U(\omega)=e^{i \omega^{\alpha} T_{\alpha}} \quad \alpha=0, \ldots, 3 \\
& T_{0}=\mathbb{I} \quad T_{1}=\gamma^{5} \quad T_{2}=\frac{1}{i} \gamma^{3} \quad T_{3}=\gamma^{3} \gamma^{5} .
\end{aligned}
$$

The mass term $m \bar{\psi} \psi$ of the lagrangian breaks this symmetry group into the subgroup $U(1) \times U(1)$ with generators $T_{0}$ and $T_{3}$. In the limit where the mass $m$ tends to zero, the $U(2)$ symmetry is not recovered and one obtains a continuum of degenerate vacua. The result (10) can be interpreted as an effect of spin polarization (or spin orientation) due to the magnetic field. A generalization of formula (1) in which the magnetic flux $\int d^{2} x B(\mathbf{x})$ replaces $B$ is valid in the inhomogeneous case [10, 6].

The problem of a free Dirac field, minimally coupled to a homogeneous magnetic field, can be exactly solved and in the Landau gauge the expression of the Dirac field in the chiral version is $(e B>0)$ :

$$
\begin{gathered}
\psi^{B}(x)=\left(\begin{array}{c}
\psi_{1}^{B}(x) \\
\psi_{2}^{B}(x)
\end{array}\right) \\
\psi_{1}^{B}(x)=\sum_{n=0}^{\infty} \sum_{p^{1}}\left\{u_{n p^{1}}(x) a_{n p^{1}}+v_{n-p^{1}}(x) b_{n p^{1}}^{\dagger}\right\} \\
\psi_{2}^{B}(x)=\sum_{n=0}^{\infty} \sum_{p^{1}}\left\{u_{n p^{1}}^{(2)}(x) c_{n p^{1}}+v_{n-p^{1}}^{(2)}(x) d_{n p^{1}}^{\dagger}\right\} \\
u_{n p^{1}}(x)=\frac{1}{\sqrt{l L_{1}}} e^{-i E_{n} t} e^{i p^{1} x^{1}}\left(\begin{array}{c}
A_{n} w_{n}\left(\xi_{x^{2}}^{p^{1}}\right) \\
-i B_{n} w_{n-1}\left(\xi_{x^{2}}^{p^{1}}\right)
\end{array}\right) \\
v_{n p^{1}}(x)=\frac{1}{\sqrt{l L_{1}}} e^{+i E_{n} t} e^{i p^{1} x^{1}}\left(\begin{array}{c}
B_{n} w_{n}\left(\xi_{x^{2}}^{p^{1}}\right) \\
+i A_{n} w_{n-1}\left(\xi_{x^{2}}^{p^{1}}\right)
\end{array}\right) \\
u_{n p^{1}}^{(2)}(x)=(-1)^{n} v_{n-p^{1}}(-x) \quad v_{n p^{1}}^{(2)}(x)=(-1)^{n} u_{n-p^{1}}(-x)
\end{gathered}
$$




$$
\begin{gathered}
A_{n}=\sqrt{\frac{E_{n}+m}{2 E_{n}}} \quad B_{n}=\sqrt{\frac{E_{n}-m}{2 E_{n}}} \\
E_{n}=\sqrt{m^{2}+2 n e B} \quad \xi_{x^{2}}^{p^{1}}=\frac{x^{2}}{l}+l p^{1}=\sqrt{e B} x^{2}+\frac{p^{1}}{\sqrt{e B}} \\
w_{n}(\xi)=c_{n} e^{-\xi^{2} / 2} H_{n}(\xi)=\left(2^{n} n ! \sqrt{\pi}\right)^{-1 / 2} e^{-\xi^{2} / 2} H_{n}(\xi) .
\end{gathered}
$$

The operators $a_{n p^{1}}, \ldots, d_{n p^{1}}$ satisfy the usual canonical anticommutation relations and $H_{n}(\xi)$ are the Hermite polynomials. The case $e B<0$ can be obtained by applying the charge conjugation operator.

One of the main properties of the theory in the minimal versions is the spectrum asymmetry concerning the lowest Landau level (LLL). This property holds also for an inhomogeneous magnetic field [1, 8, 13]. For example, if $m>0$ (that is in the representation (5)), and if $e B>0$, the energy spectrum is described by:

$$
E= \pm \sqrt{m^{2}+(2 n+1-\sigma) e B} \quad \begin{cases}\sigma=+1 & \text { if } E>0 \\ \sigma=-1 & \text { if } E<0\end{cases}
$$

where $(1 / 2) \sigma$ are the eigenvalues of the spin operator $S_{3}=(1 / 2) \sigma_{3}$. From (16) $u_{0 p^{1}} \neq 0$ and $v_{0 p^{1}} \equiv 0$. The situation is inverted in the representation (6) $(m<0)$.

\section{Vacuum state}

The lagrangian, describing the Dirac field in presence of an external magnetic field, is bilinear in the fermionic field $\psi^{B}(x)$, and we may expect that the vacuum state of the theory can be calculated by an approach " à la Bogoliubov", that is by relating the fermionic field in the presence of a magnetic field to the free field by means of a linear transformation. The calculation, which we will now illustrate, will refer to the minimal version representation (5), as the generalization to the chiral version is immediate.

The Dirac equations describing in $2+1$ dimensions the free field and the field in presence of the external field are first order differential equations, and it is possible to impose the same initial condition:

$$
\psi_{1}(0, \mathbf{x})=\psi_{1}^{B}(0, \mathbf{x}) .
$$


For the free field we have the plane waves decomposition:

$$
\begin{gathered}
\psi_{1}(x)=\sum_{\mathbf{p}} \sqrt{\frac{m}{L_{1} L_{2} E_{\mathbf{p}}}}\left\{u(\mathbf{p}) e^{-i p \cdot x} a_{\mathbf{p}}+v(\mathbf{p}) e^{i p \cdot x} b_{\mathbf{p}}^{\dagger}\right\} \\
u(\mathbf{p})=\sqrt{\frac{E_{\mathbf{p}}+m}{2 m}}\left(\frac{p^{2}-i p^{1}}{E_{\mathbf{p}}+m}\right) \quad v(\mathbf{p})=\sqrt{\frac{E_{\mathbf{p}}+m}{2 m}}\left(\begin{array}{c}
\frac{p^{2}+i p^{1}}{E_{\mathbf{p}}+m} \\
1
\end{array}\right)
\end{gathered}
$$

where $E_{\mathbf{p}}=\left(m^{2}+|\mathbf{p}|^{2}\right)^{1 / 2}$.

The initial condition (21) enables us to find the canonical transformation between the operators $\left(a_{n p^{1}}, b_{n p^{1}}\right)$ and the corresponding creation and destruction operators in absence of the magnetic field $\left(a_{\mathbf{p}}, b_{\mathbf{p}}\right)$. The free field $\psi_{1}(x)$ and the one in presence of a magnetic field $\psi_{1}^{B}(x)$ are not developed in the same bases of wave functions, and to obtain the desired canonical transformation the following relations are useful:

$$
\begin{aligned}
e^{i p^{2} x^{2}} & =e^{-i l^{2} p^{1} p^{2}} \sqrt{2 \pi} \sum_{n=0}^{\infty}(i)^{n} w_{n}\left(\xi_{x^{2}}^{p^{1}}\right) w_{n}\left(l p^{2}\right) \\
w_{n}\left(\xi_{x^{2}}^{p^{1}}\right) & =\frac{\sqrt{2 \pi}(-i)^{n} l}{L_{2}} \sum_{p^{2}} w_{n}\left(l p^{2}\right) e^{i p^{2} x^{2}} e^{i l^{2} p^{1} p^{2}} .
\end{aligned}
$$

The expression (24) follows by analytic continuation for $t \rightarrow i$ of the relation [14]:

$$
\sqrt{\pi} \sum_{n=0}^{\infty} t^{n} w_{n}(x) w_{n}(y)=\frac{1}{\sqrt{1-t^{2}}} e^{\frac{x^{2}-y^{2}}{2}-\frac{(x-y t)^{2}}{1-t^{2}}} \quad|t|<1
$$

whereas (25) represents the Fourier transformation of a Hermite function.

Using (24) and identifying the coefficients associated to any function $w_{n}(\xi)$, we obtain the transformation

$$
\begin{gathered}
\left\{\begin{aligned}
a_{n p^{1}} & =\sum_{p^{2}} \frac{(i)^{n} D_{\mathbf{p}}}{\sqrt{L_{2}}}\left\{\alpha_{n \mathbf{p}} a_{\mathbf{p}}+\beta_{n \mathbf{p}} b_{-\mathbf{p}}^{\dagger}\right\} \\
b_{n-p^{1}}^{\dagger}= & \sum_{p^{2}} \frac{(i)^{n} D_{\mathbf{p}}}{\sqrt{L_{2}}}\left\{\delta_{n-\mathbf{p}}^{*} a_{\mathbf{p}}+\gamma_{n-\mathbf{p}}^{*} b_{-\mathbf{p}}^{\dagger}\right\} \\
\alpha_{n \mathbf{p}} & =A_{n} w_{n}\left(l p^{2}\right)+B_{n} C_{\mathbf{p}}^{*} w_{n-1}\left(l p^{2}\right) \\
\beta_{n \mathbf{p}} & =-A_{n} C_{\mathbf{p}} w_{n}\left(l p^{2}\right)+B_{n} w_{n-1}\left(l p^{2}\right) \\
\gamma_{n \mathbf{p}} & =B_{n} C_{\mathbf{p}}^{*} w_{n}\left(-l p^{2}\right)-A_{n} w_{n-1}\left(-l p^{2}\right) \\
\delta_{n \mathbf{p}} & =B_{n} w_{n}\left(-l p^{2}\right)+A_{n} C_{\mathbf{p}} w_{n-1}\left(-l p^{2}\right)
\end{aligned}\right.
\end{gathered}
$$




$$
C_{\mathbf{p}}=\frac{p^{2}+i p^{1}}{E_{\mathbf{p}}+m} \quad D_{\mathbf{p}}=\sqrt{2 \pi l} e^{-i l^{2} p^{1} p^{2}} \sqrt{\frac{E_{\mathbf{p}}+m}{2 E_{\mathbf{p}}}} .
$$

These transformations preserve the canonical anticommutation relations.

We look for a normalized state $|B\rangle$ such that:

$$
a_{n p^{1}}|B\rangle=0=b_{n p^{1}}|B\rangle \quad \forall n, p^{1}
$$

Introducing the auxiliary creation and destruction operators

$$
\begin{gathered}
\widehat{a}_{n p^{1}} \equiv \sum_{p^{2}} \frac{(i)^{n} D_{\mathbf{p}}}{\sqrt{L_{2}}} w_{n}\left(l p^{2}\right)\left\{a_{\mathbf{p}}-C_{\mathbf{p}} b_{-\mathbf{p}}^{\dagger}\right\} \\
\widehat{b}_{n-p^{1}} \equiv \sum_{p^{2}} \frac{-(i)^{n} D_{\mathbf{p}}^{*}}{\sqrt{L_{2}}} w_{n-1}\left(l p^{2}\right)\left\{C_{\mathbf{p}} a_{\mathbf{p}}^{\dagger}+b_{-\mathbf{p}}\right\}
\end{gathered}
$$

the transformation (27) can be written:

$$
\left\{\begin{array}{c}
a_{n p^{1}}=A_{n} \widehat{a}_{n p^{1}}-B_{n} \widehat{b}_{n-p^{1}}^{\dagger} \\
b_{n-p^{1}}=A_{n} \widehat{b}_{n-p^{1}}+B_{n} \widehat{a}_{n p^{1}}^{\dagger} .
\end{array}\right.
$$

with $A_{n}$ and $B_{n}$ given in (18). If $\widehat{|0\rangle}$ is the vacuum associated with the auxiliary operators

$$
\widehat{a}_{n p^{1}} \widehat{|0\rangle}=0=\widehat{b}_{n-p^{1}} \widehat{|0\rangle} \quad \forall n, p^{1}
$$

we deduce immediately the relation between $|B\rangle$ and $\widehat{|0\rangle}$ :

$$
|B\rangle=\prod_{n \geq 1} \prod_{p^{1}}\left(A_{n}+B_{n} \widehat{a}_{n p^{1}}^{\dagger} \widehat{b}_{n-p^{1}}^{\dagger}\right) \widehat{|0\rangle} .
$$

The last step consists in finding the relation between the vacuum $\widehat{|0\rangle}$ and the vacuum $|0\rangle$ in absence of magnetic field. We find easily using (31)

$$
\widehat{|0\rangle}=\prod_{\mathbf{p}} \sqrt{\frac{E_{\mathbf{p}}+m}{2 E_{\mathbf{p}}}}\left(1+C_{\mathbf{p}} a_{\mathbf{p}}^{\dagger} b_{-\mathbf{p}}^{\dagger}\right)|0\rangle .
$$

The final result is:

$$
|B\rangle=\prod_{n \geq 1 p^{1}}\left(A_{n}+B_{n} \widehat{a}_{n p^{1}}^{\dagger} \widehat{b}_{n-p^{1}}^{\dagger}\right) \prod_{\mathbf{h}} \sqrt{\frac{E_{\mathbf{h}}+m}{2 E_{\mathbf{h}}}}\left(1+C_{\mathbf{h}} a_{\mathbf{h}}^{\dagger} b_{-\mathbf{h}}^{\dagger}\right)|0\rangle .
$$


The independence of $\widehat{|0\rangle}$ on the magnetic field is a consequence of the fact that in (31) $B$ appears only in the multiplicative factors of the Bogoliubov transformations within curly brackets. It is clear that the structure of the vacuum does not change when $m \rightarrow 0$.

In order to clarify the meaning of the auxiliary operators let us write the hamiltonian in presence of magnetic field in terms of these operators. Using (31) we find

$$
\begin{aligned}
\mathrm{H}^{B} & =\widehat{\mathrm{H}}-\sum_{n=1}^{\infty} \sum_{p^{1}} \sqrt{2 n e B}\left(\widehat{a}_{n p^{1}}^{\dagger} \widehat{b}_{n-p^{1}}^{\dagger}+\widehat{b}_{n-p^{1}} \widehat{a}_{n p^{1}}\right) \\
\widehat{\mathrm{H}} & \equiv m \sum_{n=0}^{\infty} \sum_{p^{1}}\left(\widehat{a}_{n p^{1}}^{\dagger} \widehat{a}_{n p^{1}}+\widehat{b}_{n p^{1}}^{\dagger} \widehat{b}_{n p^{1}}\right) .
\end{aligned}
$$

The expression of the hamiltonian $\widehat{\mathrm{H}}$ shows that $\widehat{a}_{n p^{1}}$ and $\widehat{b}_{n p^{1}}$ describe particles with degenerate energy $m$, that is the energy of the LLL. The magnetic field induces creation and destruction of particle-antiparticle pairs of the auxiliary field and this removes the degeneracy giving the usual Landau levels.

The auxiliary field $\widehat{\psi}(x)$ is

$$
\widehat{\psi}(x) \equiv \sum_{n p^{1}}\left\{\widehat{u}_{n p^{1}}(x) \widehat{a}_{n p^{1}}+\widehat{v}_{n-p^{1}}(x) \widehat{b}_{n p^{1}}^{\dagger}\right\}
$$

with

$$
\begin{aligned}
& \widehat{u}_{n p^{1}}(x)=\frac{1}{\sqrt{l L_{1}}} e^{-i m t} e^{i p^{1} x^{1}} w_{n}\left(\xi_{x^{2}}^{p^{1}}\right)\left(\begin{array}{l}
1 \\
0
\end{array}\right) \\
& \widehat{v}_{n p^{1}}(x)=\frac{i}{\sqrt{l L_{1}}} e^{i m t} e^{i p^{1} x^{1}} w_{n-1}\left(\xi_{x^{2}}^{p^{1}}\right)\left(\begin{array}{l}
0 \\
1
\end{array}\right)
\end{aligned}
$$

Finally the expression of the vacuum in the chiral version is

$$
\begin{aligned}
|B\rangle=\prod_{n \geq 1} \prod_{p^{1}}\left(A_{n}+B_{n} \widehat{a}_{n p^{1}}^{\dagger} \widehat{b}_{n-p^{1}}^{\dagger}\right)\left(A_{n}-B_{n} \widehat{c}_{n p^{1}}^{\dagger} \widehat{d}_{n-p^{1}}^{\dagger}\right) \cdot \\
\cdot \prod_{\mathbf{h}} \frac{E_{\mathbf{h}}+m}{2 E_{\mathbf{h}}}\left(1+C_{\mathbf{h}} a_{\mathbf{h}}^{\dagger} b_{-\mathbf{h}}^{\dagger}\right)\left(1+C_{\mathbf{h}}^{*} c_{\mathbf{h}}^{\dagger} d_{-\mathbf{h}}^{\dagger}\right)|0\rangle .
\end{aligned}
$$

When $m \rightarrow \infty, A_{n} \rightarrow 1, B_{n} \rightarrow 0, C_{\mathbf{h}} \rightarrow 0$ and $|B\rangle \rightarrow|0\rangle$. 


\section{References}

[1] Y. Aharonov and A. Casher, Phys. Rev. A. 19, 2461 (1979).

[2] T. W. Appelquist, M. Bowick, D. Karabali and L. C. R. Wijewardhana, Phys. Rev. D. 33, 3740 (1986).

[3] B. Binegar, J. Math. Phys. 23, 1511 (1982).

[4] D. Boyanovsky, R. Blankenbecler and R. Yahalom, Nucl. Phys. B270, 483 (1986).

[5] A. Chodos, K. Everding and D. A. Owen, Phys. Rew. D. 42, 2881 (1990).

[6] G. Dunne and T. Hall, Phys. Rev. D. 53, 2220 (1996).

[7] V. P. Gusynin, V. A. Miransky and I. A. Shovkovy, Phys. Rev. D. 52, 4718 (1995); Phys. Rev. D. 52, 4747 (1995); Nucl. Phys. B462, 249 (1996).

[8] R. Jackiw, Phys. Rev. D. 29, 2375 (1984).

[9] I. V. Krive, S. A. Naftulin, Sov. J. Nucl. Phys. 54, 897 (1991); Phys. Rev. D. 46, 2737 (1992).

[10] R. R. Parwani, Phys. Lett. B. 358, 101 (1995); Int. J. Mod. Phys. A. 11, 1715 (1996).

[11] J. Schwinger, Phys. Rev. 82, 664 (1951).

[12] Shuryak, E. (1995). The QCD Vacuum, Chiral Phase Transition and Quark-Gluon Plasma. In Quark-Gluon Plasma 2. (Ed. Hwa, R. C.). World Scientific, Singapore.

[13] Thaller, B. (1992). The Dirac Equation. Springer-Verlag, Berlin.

[14] Titchmarsh, E. C. (1948). Introduction to the Theory of Fourier Integrals. Claredon Press, Oxford. 